\title{
A Novel Hypomorphic CSF1R Gene Mutation in the Biallelic State Leading to Fatal Childhood Neurodegeneration
}

\author{
Parag Mohan Tamhankar ${ }^{1}$ Bin Zhu ${ }^{2}$ Vasundhara Parag Tamhankar ${ }^{1}$ Shilpa Mithbawkar ${ }^{1}$ \\ Luis Seabra $^{3}$ John H. Livingston ${ }^{4}$ Takeshi Ikeuchi ${ }^{2}$ Yanick J. Crow ${ }^{3,5}$ \\ ${ }^{1}$ Centre for Medical Genetics, Mulund, Mumbai, India \\ 2 Department of Molecular Genetics, Brain Research Institute, Niigata \\ University, Japan \\ 3 Paris Descartes University, Sorbonne-Paris-Cité, Institut Imagine, \\ Paris, France \\ ${ }^{4}$ Department of Paediatric Neurology, Leeds Teaching Hospitals and \\ University of Leeds, Leeds, United Kingdom \\ ${ }^{5}$ Centre for Genomic and Experimental Medicine, MRC Institute of \\ Genetics and Molecular Medicine, University of Edinburgh, \\ Edinburgh, United Kingdom \\ Address for correspondence Dr Parag Tamhankar, DM (Medical \\ Genetics), MD (Pediatrics), MBBS, Centre for Medical Genetics, \\ Mulund, Mumbai, India (e-mail: paragtmd2@gmail.com).
}

Neuropediatrics 2020;51:302-306.

\begin{abstract}
Keywords

- CSF1R

- adult onset leukoencephalopathy with axonal spheroids and pigmented glia

- recessive

- hypomorph

- biallelic

- India

We report the clinical and molecular characterization of a novel biallelic mutation in the CSF1R gene leading to an autosomal recessive form of childhood onset leukoencephalopathy in a consanguineous family. The female child experienced acute encephalopathy at the age of 2 years, followed by spasticity and loss of all achieved milestones over 6 months. Her elder brother presented with encephalopathy at 4 years of age, with a subsequent loss of all achieved milestones over 8 months. Brain imaging in both children revealed multiple welldefined areas of calcification in the parietal and frontal regions and the occipital horns of both lateral ventricles. Clinical exome trio analysis showed homozygosity for a p.T833M mutation in CSF1R in the girl. Heterozygous family members, including both parents, were asymptomatic, with the eldest being 68 years of age. Total CSF1R protein expression levels were normal as compared with wild-type allele, but CSF1 ligand dependent autophosphorylation was consistent with a hypomorphic allele.
\end{abstract}

\section{Introduction}

Mutations in CSF1R (colony stimulating factor 1 receptor) are a well-recognized cause of autosomal dominant leukoencephalopathy with axonal spheroids and pigmented glia (ALSP). More recently, autosomal recessive mutations in the same gene have been described to underlie a novel phenotype encompassing pediatric-onset progressive brain disease and osteopetrosis. ${ }^{1,2}$ We now report two siblings with childhood-onset neurodegeneration after an initial period of normal development likely due to a novel homozygous missense mutation in CSF1R.

\section{Clinical Data}

The patients were born to a consanguineous couple of Indian ancestry ( - Fig. 1A). Limited clinical history was available to us, but the female proband was described to have demonstrated completely normal development until the age of 2 years when she experienced an abrupt onset of encephalopathy followed by rapid loss of all achieved milestones (first motor-adaptive followed by speech) over 6 months ( - Figs. 1B-D). At the age of 3 years 11 months, she had a head circumference of $47 \mathrm{~cm}$ $(-1.59 S D)$, length of $87 \mathrm{~cm}$ ( $<$ third centile), and weight of

(c) 2020 Georg Thieme Verlag KG Stuttgart - New York
DOI https://doi.org/ 10.1055/s-0040-1702161. ISSN 0174-304X. 


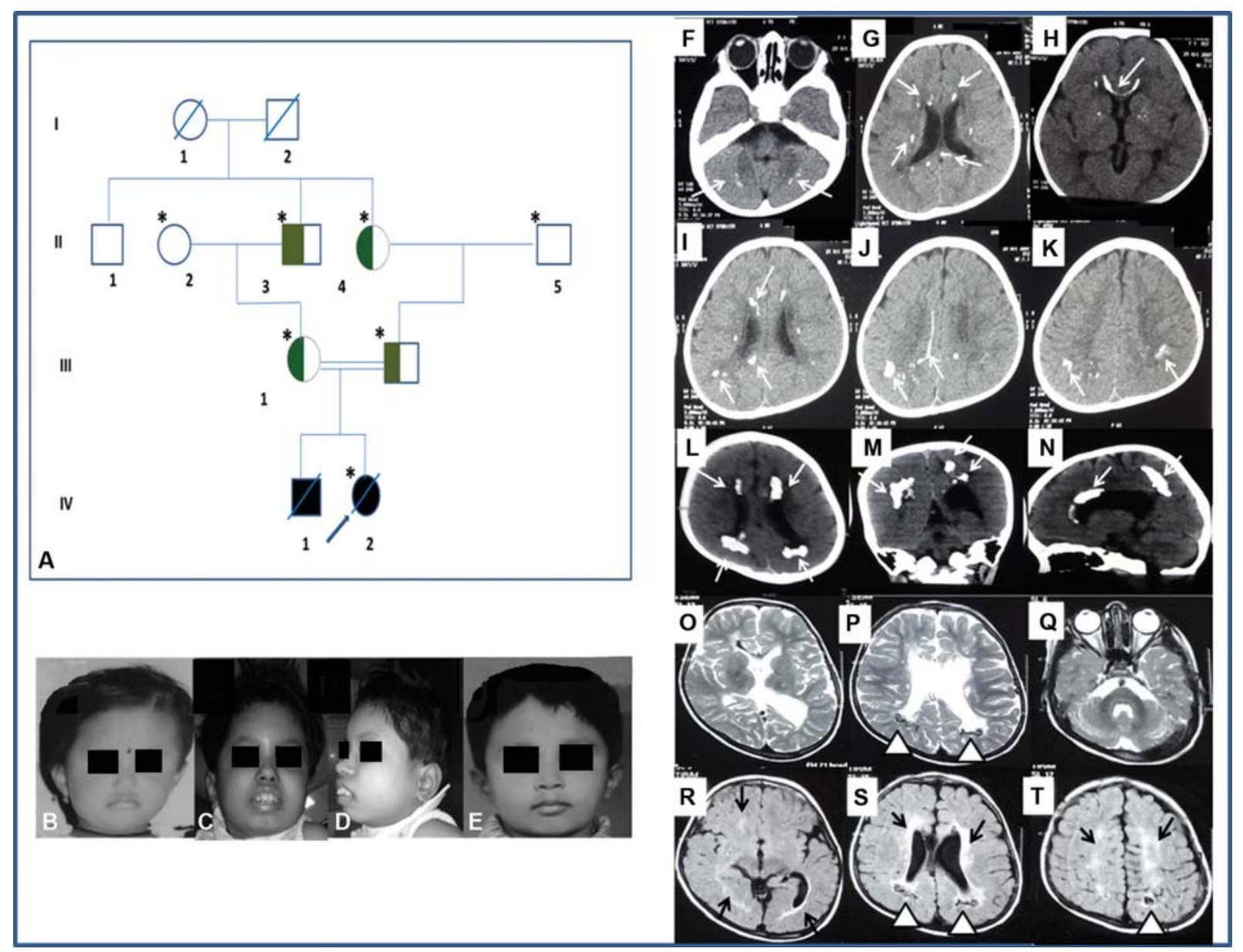

Fig. 1 (A) The pedigree of the family. IV-2 is the proband (indicated by the arrow), who died at 12 years of age; IV-1 is her elder brother who died aged 4 years 8 months (black coloring indicates clinically affected). The parents of the children are consanguineous and carriers for the $p$. Thr833Met mutation (indicated by half coloring). They are clinically healthy. II-3 (age 68 years) and II-4 (age 60 years) were found to be healthy carriers for p.Thr833Met mutation. II-3 had a normal CT scan brain. An asterisk above the pedigree symbol sign indicates individuals who underwent DNA testing. The individual I-1 reportedly died of stroke at 65 years of age; details were not available. Individual I-2 died at 95 years of age. (B-E) Clinical image of the affected siblings. Proband IV-2 at age of 2 years (B) (before onset of illness) and at age of 12 years (C, D) showing microcephaly, tall forehead, coarse facies, arched eyebrows, depressed nasal bridge, large protruding teeth and low set ears, (E) probands' elder brother IV-1 at 4 years of age again before the onset of illness (B), showing normal facial features. CT scan brain of the proband showing punctate to confluent calcifications (white arrows) in white matter of the brain (F-K). Cranial CT scan of the elder brother at age of 4 years demonstrating punctate to confluent calcifications (red arrows) in white matter of brain and cerebellum (L-N). MRI brain of the brother at age of 4 years (T-spinecho sequences: (O-Q) inversion recovery sequences: $(R-T)$ showed diffuse periventricular T2 hyperintensities (black arrows) with focal hypointensities (arrowheads) suggesting calcification. Calcification is also seen in the corpus callosum. There is loss of periventricular white matter and thinning of the corpus callosum. Brainstem, cerebral sulci, and screening for spinal cord were normal for the sibs. Informed consent for the publication of clinical pictures of the children has been obtained. CT, computed tomography.

$10.74 \mathrm{~kg}$ (<third centile). A further assessment at the age of 12 years revealed microcephaly (exact size not recorded), and she was unable to speak, communicate, visually fixate, recognize parents, control bowel/bladder, or use her hands. She had upper limb hypotonia, lower limb spasticity with contractures at the knees and ankles, brisk reflexes and extensor plantars, and she demonstrated intermittent dyskinesias. Cranial computerized tomography (CT) imaging at 2 years of age was characterized by punctate to confluent brain calcifications in the white matter (corpus callosum, centrum semiovale, subcortical white matter, and cerebellum; -Figs. 1F-K). She died shortly thereafter of pneumonia. Autopsy was not undertaken. An older male sibling to the proband demonstrated normal development until the age of 4 years ( - Fig. 1E) and then began to toe-walk reportedly due to pain in his legs. One month later, he had a febrile convulsion with brief unconsciousness after which he lost the ability to walk and speech deteriorated. Over a period of 8 months, rigidity and dyskinesia increased and he lost all motor, adaptive, speech, and social abilities. He died at 4 years 8 months of age apparently due to pneumonia. Cranial CT scan revealed multiple well-defined areas of calcification in the parietal and frontal regions and the occipital horns of both lateral ventricles (- Figs. 1L-N), while cranial magnetic resonance imaging showed cerebral atrophy with patchy areas of increased signal intensity in the white matter and ex-vacuo dilatation of the ventricles (-Figs. 10-T). Chest $X$-ray was unremarkable. Leucocyte aryl sulphatase $A$ and $\beta$ galactosidase were normal. Cerebrospinal (CSF) studies were normal including normal levels of 5 hydroxy indole acetic acid, homovanillic acid, 3,4-dihyroxyphenylacetic acid, 3-methoxy- 
4-hydroxyphenyl glycole, 3-o-methyl dopa, 5-hydroxytryptophan, 5-methyl tetrahydrofolic acid, neopterin, and biopterin. Brain biopsy of the left frontal lobe was performed on the boy and was reported as inconclusive on cytopathology, with absence of toxoplasma and cytomegalovirus antigen on immunohistochemistry. Staining for microglia was not undertaken. The important clinical differential of Aicardi-Goutières syndrome was considered and made less likely by the absence of a CSF lymphocytosis, normal CSF levels of neopterin and biopterin, as well as a difference in the pattern of calcification seen.

\section{Genetic and Functional Data}

Trio whole exome sequencing identified the female child to be homozygous for a missense mutation (Chr5:149435645G > A /c.2498C $>$ T /p.(Thr833Met)/rs780804532) in CSF1R. The child's mother and father, aged 35 and 41 years of age respectively, were heterozygous for this variant. No sample was available from the deceased brother. The c.2498C $>$ T transition was also present in the heterozygous state in the maternal grandfather and paternal grandmother (siblings to one another), both of whom were healthy at the ages of 68 and 60 years respectively, with a cranial $\mathrm{CT}$ in the former being completely unremarkable at this age. The putative mutant allele is only recorded once, in the heterozygous state, out of 251,366 alleles on gnomAD. It has additionally been incidentally reported in two adult carriers without neurological disease. ${ }^{3}$ The variant is predicted to be pathogenic by Protein Variant Effect Analyzer (PROVEAN), Polyphen2 and MutationTaster software, and is conserved in evolution across multiple species to zebrafish. ${ }^{4-6}$ Using American Council of Medical Genetics guidelines, the $\mathrm{p}$. (Thr833Met) substitution is classified as likely pathogenic (IIIB) (PS3, PM1, PM2, PP3, and PP4 criteria). ${ }^{7}$ The threonine at position 833 falls within the tyrosine kinase domain (TKD; amino acid residues 582-910) of the encoded protein, a mutational hotspot with multiple unique pathogenic alleles reported in the context of ALSP. ${ }^{8}$ Analysis by HOPE software showed that mutant residue is bigger and more hydrophobic than wildtype residue. The wild-type residue normally forms a hydrogen bond with glutamine 835; the mutant residue may affect this bond formation. The residue at 833 is located on surface of the protein and mutation at this residue can disturb interactions with other molecules or other parts of the protein (-Supplementary File, -Supplementary Animation S1, and -Supplementary Animation S2; online only). ${ }^{9}$ The p. (Thr833Met) observed in our family was further evaluated by transfecting HEK293T cell lines followed by immunoblot analysis and functional test of CSF1R ligand dependent autophosphorylation (LDA) at important tyrosine residues. ${ }^{10}$ This analysis demonstrated that the total CSF1R protein expression levels were normal. But the autophosphorylation was completely lost at the p.Ty546, and p.Tyr708 positions, while the percentage of autophosphorylation (LDA) at p.Tyr723 was $22 \%$ of wild-type levels (-Figs. 2A-C).

\section{Discussion}

Patients with autosomal dominant ALSP typically demonstrate progressive neurodegeneration, with mean age at onset as 43 years, mean duration of disease being 6.8 years, range of presenting ages being 18 to 78 years. ${ }^{11}$ The disease is characterized by a complex phenotype including progressive dementia, apraxia, apathy, ataxia, parkinsonism, spasticity, and epilepsy. ${ }^{8}$ Brain imaging is characterized by intracranial calcification, multifocal white matter demyelination, cerebral atrophy $8,10,12$ while brain biopsy shows loss of myelin sheaths and axons, spheroids, and pigmented macrophages.
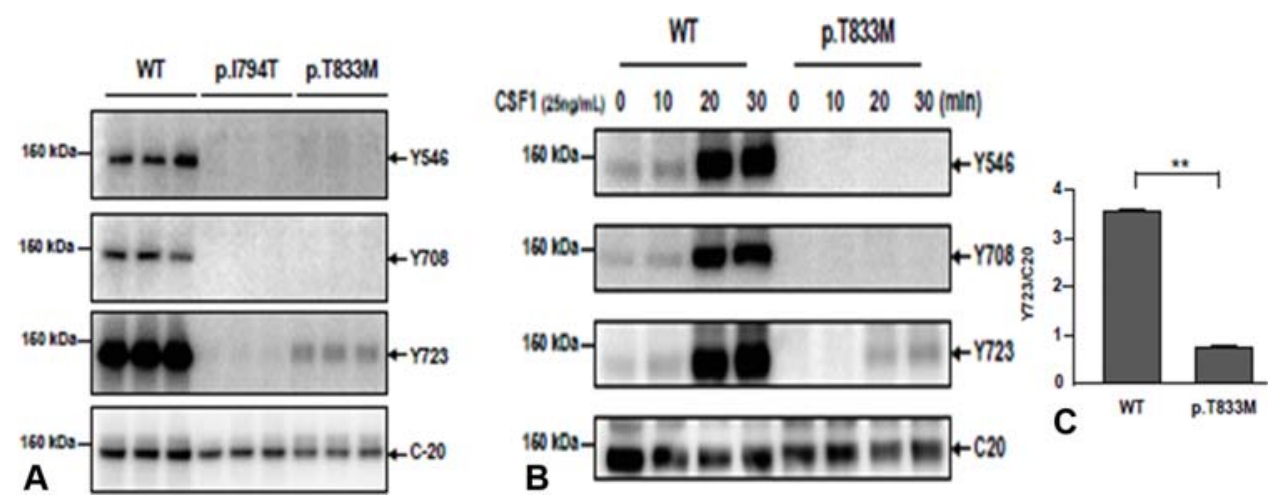

Fig. 2 Functional assay of mutant CSF1Rs. (A) Western blot analysis: HEK293T cells were transiently transfected with wild-type or mutant CSF1R and stimulated with its ligand CSF1 to induce autophosphorylation. Detergent cell lysates were subjected to immunoblot analysis using either specific antiphosphorylated CSF1R antibodies. (antiphosphorylated-Y546-CSF1r (first/top panel); antiphosphorylated-Y708-CSF1R [second panel]; antiphosphorylated-Y723-CSF1R [third panel]) OR antiantibody for C-terminus C-20 (bottom panel) to detect total CSF1R. The assay shows normal autophosphorylation at $Y 546, Y 708$, and $Y 723$ for wild-type alleles but absent autophosphorylation at positions $Y 546, Y 708$, Y723 for alleles p.1794T and whereas for p.T833M, autophosphorylation at positions Y546, Y708 is absent but partially present at Y723 position. The total protein in both wild-type and mutant alleles is comparable (bottom panel). (B) Ligand-dependent autophosphorylation of CSF1R was examined in cells transiently transfected with the wild-type or variant CSF1Rs. Detergent-extracted lysates were collected at the indicated time (min) after CSF1 stimulation. Increased levels of phosphorylated CSF1R were observed in cells expressing the wild-type allele but results for $\mathrm{p}$. T833M allele showed absent at Y546, Y708 positions and partially present at Y723 position. (C) Results of semiquantification of immunoblot data: the mean intensity of autophosphorylation at Y723 position of CSF1R was normalized to the total amount of CSF1R. Mean data are presented. The mean intensity for p.T833M allele is $22 \%$ that of wild allele. 
Intracranial calcification can occur since birth and is independent of white matter degeneration or clinical symptoms. Since the original discovery as causative for hereditary leukoencephalopathy (ALSP) in 2012, several families have been described with 102 unique mutations listed in Human Gene Mutation Database. Almost all mutations are located in tyrosine kinase domain of CSF1R protein. ${ }^{13}$

The first reported recessive cases (year 2017) include two Arab sibs, with osteopetrosis, hypocalcemia, agenesis of the corpus callosum, enlarged ventricles, periventricular calcifications, cerebellar hypoplasia leading to perinatal lethality. DNA tests of children were not available, but the consanguineous parents were carriers for p.Y540X mutation in CSF1R gene. ${ }^{14}$ Another infant from Alaska, (year 2019) homozygous for c.1754-1G > C(p.Gly585_Lys619delinsAla) presented with Dandy-Walker syndrome, agenesis of corpus callosum, ventriculomegaly, osteopetrosis, hypocalcemia, epilepsy, swallowing difficulties, death at 10 months due to septicaemia. ${ }^{2}$ Another Arab patient with homozygosity for c.1929C $>$ A (p.His643Gln) was 24 years old with leukodystrophy, pontocerebellar hypoplasia, periventricular calcification, and onset of neuroregression since 12 years of age. ${ }^{2}$ This patient is similar to our patients in having a period of normal development before the onset of disease. Several of the patients described by Guo et al, including a 37-year-old woman who was diagnosed with osteopetrosis at age of 5 years, but did not experience any neurological symptoms until the third decade of life. ${ }^{1,2}$ Dysmorphic facial features, such as long eyelashes, epicanthic folds, bulbous nose, dysplastic ears, and macrocephaly $(+5 S D)$, have also been described in recessive form. ${ }^{1,2}$ Patients with biallelic mutations can also have osteosclerosis of the craniofacial bones and skull, platyspondyly and abnormalities of the long bones, including undermodeling, widened metaphyses, and constricted and sclerotic diaphyses. Mild facial dysmorphism but no skeletal findings were present in our cases. Thus, biallelic mutations in CSF1R are associated with a remarkably broad phenotypic and neuroradiological spectrum.

The CSF1R protein is composed of extracellular ligand binding domain, a transmembrane domain and an intracellular tyrosine kinase domain (TKD). The binding of ligand CSF1 to CSF1R results in autophosphorylation of several tyrosine residues in the TKD followed by phosphorylation of downstream effector proteins. The molecular mechanism of CSF1R gene pathology is not exactly understood. It is intriguing to note that mutations leading to dominant form of disease can have haploinsufficiency, dominant negative, or gain-of-function mechanisms. Heterozygosity for ALSP causative mutation p.P104Lfs* 8 (exon 4 , much before the TKD) causes haploinsufficiency due to nonsense mediated decay of the mRNA from mutant allele. ${ }^{10}$ In contrast, the mutation p.Y886Qfs*55 (exon 21, TKD) causes truncated protein with absent autophosphorylation and causes dominant negative mechanism by forming heterodimers with wild-type chain. ${ }^{10}$ Gain-of-function mutations such as K584E, R802V, T633 (gatekeeper) residue mutations led to constitutive activation (ligand independent autophosphor- ylation) and subsequent rapid degradation ultimately leading to decreased protein levels (haploinsufficiency). ${ }^{11}$ Homozygosity for amorphic alleles (e.g., c.1754-1G > C, p. $\mathrm{Y}_{540^{*}}$ ) have been reported to lead to perinatal death but in these families, the carriers (parents and/or grandparents) were not old enough to have excluded presentation as ALSP later in life. ${ }^{2}$ These could be examples of severe presentation in patients homozygous for dominant alleles. However, mutations leading to "true" recessive form of disease are partial loss of function type (hypomorphic). This suggestion is further strengthened by our description of individuals heterozygous for the p.Thr833Met (LDA 22\% of wild type) who remains asymptomatic in the seventh decade of life, with normal neuroimaging recorded in one. Previously described recessive alleles include hypomorphs p.Pro132Leu (LDA 50\%), p.Lys627del (LDA 40\%), p.Ser620delins40 (LDA $15 \%$ ), and p.His643Gln (LDA not available). Compound heterozygosity for hypomorphs (p.Lys627del and p.Ser620delins40) led to osteopetrosis-related fracture at 5 years but neurological disease at 33 years. Compound heterozygosity for amorph (p.Gln481*) and hypomorph (p.Pro132Leu) led to Dandy-Walker syndrome and hydrocephalus requiring shunt at the age of 3 months with carrier adults having mild bone disease and dementia at 70 years. ${ }^{1}$ Homozygosity for hypomorphic allele c.1969+115_1969+116del in intron 14 that affects a splicing regulatory site led to varying degrees of intellectual disability followed by childhood onset progressive encephalopathy at 14 to 23 years and milder bone disease. The carrier parents aged 70 and 69 years were normal clinically and on neuroimaging; thus, these alleles are also true recessive. ${ }^{1}$

In conclusion, we identify a novel biallelic mutation in CSF1R associated with childhood-onset neurodegeneration and provide further functional evidence that mutations associated with CSF1R-related autosomal recessive disease likely act as hypomorphic alleles.

\section{Ethics Statement}

The study was approved by the Leeds (East) Research Ethics Committee (10/H1307/132) and the Comite de Protection des Personnes (ID-RCB/EUDRACT: 2014-A01017-40).

\section{Authors Contribution}

P.M.T., T.I. and Y.J.C. participated in the design of the study. P.M.T., B.Z., V.P.T., S.M., and L.S. collected and/or generated data. P.M.T., V.P.T., J.H.L., T.I., and Y.J.C. analyzed and interpreted the data. P.M.T., T.I., and Y.J.C. drafted the manuscript. All co-authors read and approved the final manuscript.

\section{Funding}

National Research Agency (France) under the 'Investments for the Future' program bearing the reference ANR-10-IAHU-01 and the MSDAvenir fund (DEVO-DECODE Project).

Conflict of Interests

None declared. 


\section{Acknowledgment}

We thank the family of the patients for their support for this research. We acknowledge technical help from Rutuja Dhamanaskar, Rakhi Kesarkar, Deepti Mestry, and Prajakta Nar. Y.J.C. acknowledges a state subsidy managed by the National Research Agency (France) under the "Investments for the Future" program bearing the reference ANR10-IAHU-01 and the MSDAvenir fund (DEVO-DECODE Project).

\section{References}

1 Guo L, Bertola DR, Takanohashi A, et al. Bi-allelic CSF1R mutations cause skeletal dysplasia of dysosteosclerosis-pyle disease spectrum and degenerative encephalopathy with brain malformation. Am J Hum Genet 2019;104(05):925-935

2 Oosterhof N, Chang IJ, Karimiani EG, et al. Homozygous mutations in CSF1R cause a pediatric-onset leukoencephalopathy and can result in congenital absence of microglia. Am J Hum Genet 2019; 104(05):936-947

3 Filges I, Manokhina I, Peñaherrera MS, et al. Recurrent triploidy due to a failure to complete maternal meiosis II: whole-exome sequencing reveals candidate variants. Mol Hum Reprod 2015;21 (04):339-346

4 Choi Y, Chan AP. PROVEAN web server: a tool to predict the functional effect of amino acid substitutions and indels. Bioinformatics 2015;31(16):2745-2747

5 Ramensky V, Bork P, Sunyaev S. Human non-synonymous SNPs: server and survey. Nucleic Acids Res 2002;30(17):3894-3900
6 Schwarz JM, Cooper DN, Schuelke M, Seelow D. MutationTaster2: mutation prediction for the deep-sequencing age. Nat Methods 2014;11(04):361-362

7 Kleinberger J, Maloney KA, Pollin TI, Jeng LJ. An openly available online tool for implementing the ACMG/AMP standards and guidelines for the interpretation of sequence variants. Genet Med 2016;18(11):1165

8 Konno T, Kasanuki K, Ikeuchi T, Dickson DW, Wszolek ZK. CSF1Rrelated leukoencephalopathy: A major player in primary microgliopathies. Neurology 2018;91(24):1092-1104

9 Venselaar H, Te Beek TA, Kuipers RK, Hekkelman ML, Vriend G. Protein structure analysis of mutations causing inheritable diseases. An e-Science approach with life scientist friendly interfaces. BMC Bioinformatics 2010;11:548

10 Miura T, Mezaki N, Konno T, et al. Identification and functional characterization of novel mutations including frameshift mutation in exon 4 of CSF1R in patients with adult-onset leukoencephalopathy with axonal spheroids and pigmented glia. J Neurol 2018;265(10):2415-2424

11 Lynch DS, Houlden H. Stem cell transplant arrests decline in case of CSF1R leukoencephalopathy. J Neurol Neurosurg Psychiatry 2019;90(12):1306

12 Pridans C, Sauter KA, Baer K, Kissel H, Hume DA. CSF1R mutations in hereditary diffuse leukoencephalopathy with spheroids are loss of function. Sci Rep 2013;3:3013

13 The Human Gene Mutation Database at the Institute of Medical Genetics in Cardiff. Available at: http://www.hgmd.cf.ac.uk/ac/ gene.php?gene=CSF1R. Accessed October 9, 2019

14 Monies D, Maddirevula S, Kurdi W, et al. Autozygosity reveals recessive mutations and novel mechanisms in dominant genes: implications in variant interpretation. Genet Med 2017;19(10):1144-1150 